\title{
United States - Countervailing Measures on Supercalendered Paper from Canada, DS505 - ERRATUM
}

\author{
Marcus Sohlberg
}

https://doi.org/10.1017/S1474745620000245, Published by Cambridge University Press, 8 June 2020.

In this case summary a number of text corrections were not made in the final published version. These text corrections are detailed below.

Page 479 Line 6 '... It is the USDOC's conduct in applying AFA ...' should read '...It is the USDOC's conduct to apply AFA ...'

Page 479 Line 7 '... under the OFA question that Canada ...' should read '... under the OFA question, but later discovered during investigation, that Canada ...'

Page 479 Line 14 '... that Canada had provided ...' should read '...that Canada provided ...'

Page 479 Line $37^{\text {' } . . . ~ A g r e e m e n t, ~ a n d ~ t h a t ~ a n ~ i n v e s t i g a t i n g ~ a u t h o r i t y ~ m a y ~ n o t ~ s i m p l y ~ . . . ' ~ s h o u l d ~}$ read '... Agreement, because the USDOC may not simply ...'

Page 479 Footnote 5 '... Apellate Body Report, US-Supercalendered Paper, paras. 5.44, 5.47 ...' should read '... Ibid. paras. 5.44, 5.47 ...'

Page 480 Line 6 '... determine whether missing information ...' should read '... determine whether the missing information ...'

Page 480 Line 15 '... not just any information can be missing ...' should read '... not just any information is missing ...'

Page 480 Line 24 '... Lawyers at White \& Case LLP, Geneva, Switzerland, but written in their personal capacities ...' should read '... Marcus Sohlberg. Counsel, White \& Case LLP, Geneva, Switzerland, but written in personal capacity. ...'

The publisher apologises for these errors.

\section{Reference}

M. Sohlberg, 'United States - Countervailing Measures on Supercalendered Paper from Canada, DS505', 19(3) World Trade Review (2020), 478-480. https://doi.org/10.1017/S1474745620000245

Cite this article: Sohlberg M (2020). United States - Countervailing Measures on Supercalendered Paper from Canada, DS505 - ERRATUM. World Trade Review 19, 622-622. https://doi.org/10.1017/S1474745620000476

(c) The Author(s), 2020. Published by Cambridge University Press 